\title{
Reply to "Comment on mechanical analog of temperature for the description of force distribution in static granular packings"
}

\author{
A. H. W. Ngan \\ Department of Mechanical Engineering, The University of Hong Kong, Pokfulam Road, Hong Kong, China
}

(Received 25 November 2003; published 4 May 2004)

\begin{abstract}
Metzger [preceding paper, Phys. Rev. E 69, 053301 (2004)] put forward a number of arguments against the Gaussian force distribution concluded in the investigations by Ngan [Phys. Rev. E 68, 011301 (2003)] and by some previous workers. It is shown here that, with a more proper choice for the constraint in the free energy minimization, the Shannon entropy approach can predict three-dimensional force distributions that are in much better agreement with simulated distributions. In two-dimensional packings, if one accepts a Gaussian distribution at some finite strain in a Hookean force-law situation, then the superposition principle of linear elasticity predicts that the same Gaussian distribution will be maintained at vanishing strains. This argument forms an interesting dilemma with Metzger's argument about the noninvolvement of the force law in statically determinate packings.
\end{abstract}

DOI: 10.1103/PhysRevE.69.053302

PACS number(s): 45.05.+x, 45.70. $-\mathrm{n}, 81.05 . \mathrm{Rm}, 05.70 .-\mathrm{a}$

\section{INTRODUCTION}

In his Comment [1], Metzger questions the applicability of my predicted Gaussian distribution for two dimensions (2D) and the nearly Gaussian distribution for 3D, to situations when the grain deformation is vanishingly small but when the contact forces are large so that $\langle f\rangle>3$ and 2 in 2D and $3 \mathrm{D}$, respectively $(\langle f\rangle=$ normalized contact force). He argues that the probabilities of the large forces are not calculated to a high enough precision to unambiguously verify the Gaussian or nearly-Gaussuan distribution. While I share Metzger's view on this, one must not neglect that the probabilities of small forces, which are abundant and are therefore calculated to a high precision, are well described by the Gaussian form in 2D (see Fig. 3 of Ref. [2]). In 3D, Metzger correctly pointed out that the originally predicted distribution cannot capture precisely the small and large forces at the same time. This can be seen in the semilogarithmic scale (for large forces) as shown in Fig. 7 of Ref. [2], as well as the linear scale (for small forces) as shown in Fig. 1 here. The inapplicability of the Shannon entropy as suggested by Metzger could be a reason for the inaccurate representation of the $3 \mathrm{D}$ results, but here, I first argue that a simple modification to the constraint in the free energy minimization can remove the discrepancy in the $3 \mathrm{D}$ situation to a large extent. Metzger's argument against the use of the Shannon entropy in the packing situation will be discussed in a later section.

\section{MODIFIED CONSTRAINT FOR THE FORCE PROBABILITY}

The representation of the randomness of the packing by the entropy functional implies an ensemble concept-i.e., $S$ $=k \ln w$, where $w$ is the number of different packing configurations exhibiting the same force distribution $P(f)$ and subjected to the same external pressure. The constantpressure requirement among different replicas of the en- semble should correspond to a requirement of the mean strain of the packings being constant. Depending on the force law in the system, the mean-strain constraint may or may not be equivalent to the mean-force constraint. If the force law is Hookean, as would be the case for Hertzian contacts in a 2D situation, the mean-strain constraint is equivalent to the mean-force constraint, since a Hookean force is proportional to the strain it produces. Therefore, the predicted force distribution is still the same Gaussian form as in Eq. (8) of Ref. [2]. The force normalization constant defining $\langle f\rangle$ should also be the average force

$$
\bar{f}=\int_{0}^{\infty} f P(f) d f .
$$

In 3D Hertzian contact, however, the mean-strain constraint and mean-force constraint are no longer equivalent. Using

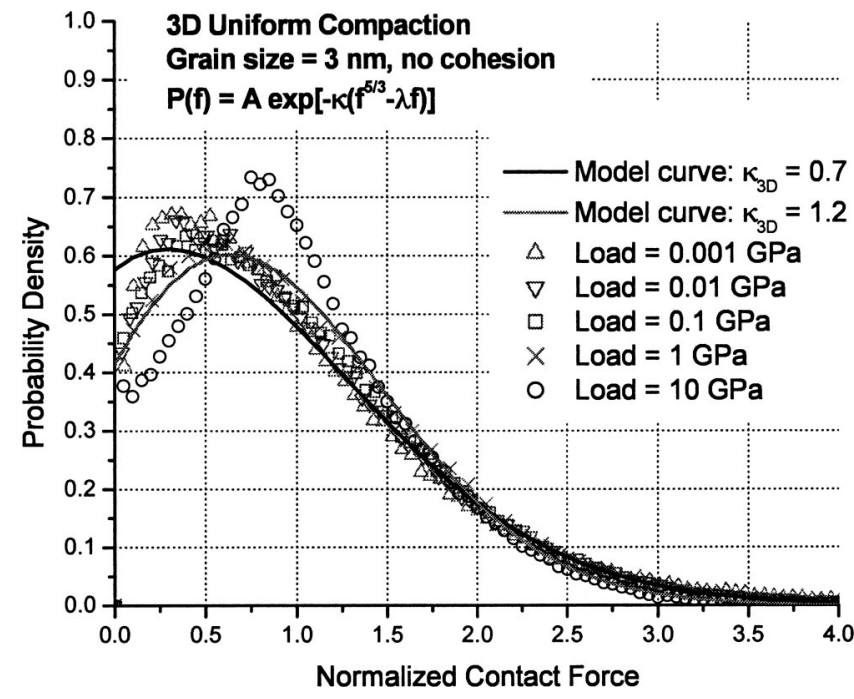

FIG. 1. Computer-simulated force distributions in 3D amorphous packings, replotted in linear scale using the same data from Fig. 7 of Ref. [2]. The discrepancy between the theoretical curves and the simulated results are apparent in the small-force range. 


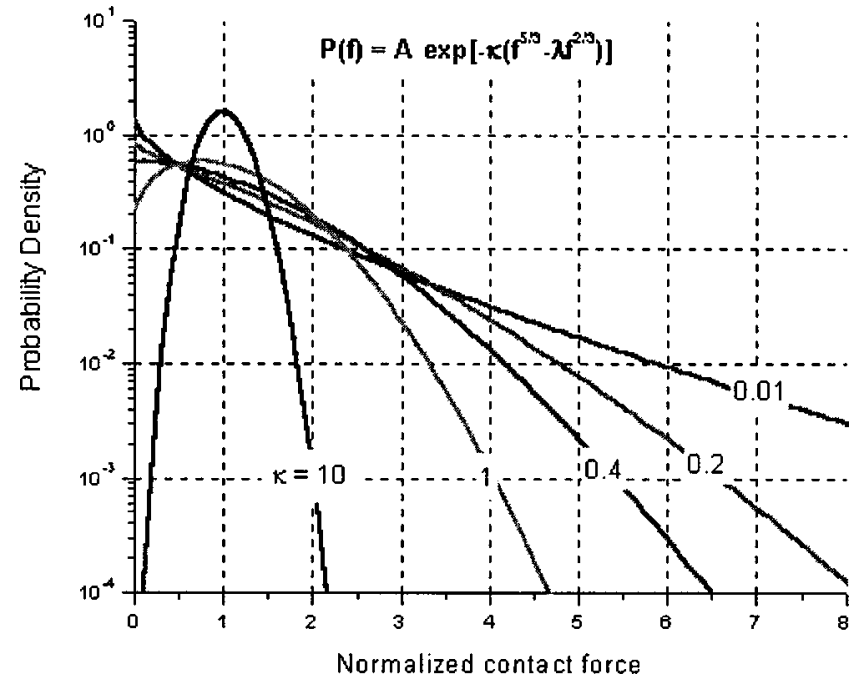

FIG. 2. Theoretical force distribution for 3D Hertzian contact using the mean-strain constraint. The normalized force in the abscissa is $\langle f\rangle=f / \hat{f}$.

the 3D Hertzian force law given in Eq. (9) of Ref. [2], the mean strain of the contact can be shown to be

$$
\begin{aligned}
\bar{\varepsilon} & =\int \frac{\delta R}{R} P(f) d f=\frac{1}{8 R^{2}}\left(\frac{3 R}{E_{r}}\right)^{2 / 3} \int f^{2 / 3} P(f) d f \\
& =\frac{1}{8 R^{2}}\left(\frac{3 R \hat{f}}{E_{r}}\right)^{2 / 3},
\end{aligned}
$$

where the notations are given in Sec. II B 1 of Ref. [2]. In Eq. (2) here, $\hat{f}=\left(\int f^{2 / 3} P(f) d f\right)^{3 / 2}$ is a geometrical mean of the contact forces which is in general different from the arithmetic mean in Eq. (1), and hence setting $\hat{f}$ constant produces different effects from setting $\bar{f}$ constant. The mean-strain constraint in the 3D situation can be shown to yield the following force distribution instead of Eq. (11) in Ref. [2]:

$$
P(f)=A \exp \left[-\kappa\left(\langle f\rangle^{5 / 3}-\lambda\langle f\rangle^{2 / 3}\right)\right] .
$$

Here, $\langle f\rangle=f / \hat{f}$ is the contact force normalized by the new constant $\hat{f}=\left(\int f^{2 / 3} P(f) d f\right)^{3 / 2}$, instead of by $\bar{f}$ as in Eq. (1). The $\kappa$ in Eq. (3) is defined in terms of the new normalization constant $\hat{f}$ as

$$
\kappa=\frac{2}{5 R}\left(\frac{3 R}{8 E_{r}}\right)^{2 / 3} \hat{f}^{5 / 3} \frac{1}{k \theta} .
$$

For any given value of $\kappa$, the normalization constants $A$ and $\lambda$ can be calculated from the constraints $\int P(\langle f\rangle) d\langle f\rangle=1$ and $\int\langle f\rangle^{2 / 3} P(\langle f\rangle) d\langle f\rangle=1$, the latter being due to $\bar{\varepsilon}$ in Eq. (2) being held constant. Figure 2 shows the force distributions predicted by Eq. (3) at different values of $\kappa$.

In Fig. 3 are replotted the simulated results in Fig. 1 in both the semilogarithmic and linear scales, and it can be seen that the simulated results at different applied pressures nearly collapse onto a single curve, showing excellent agreement with Eq. (3). Such a good agreement is the best available so far from a theoretical derivation. The only exception may be the statistics of large forces at the slightest applied pressure of $0.001 \mathrm{GPa}$. It is not clear at this stage whether the discrep-

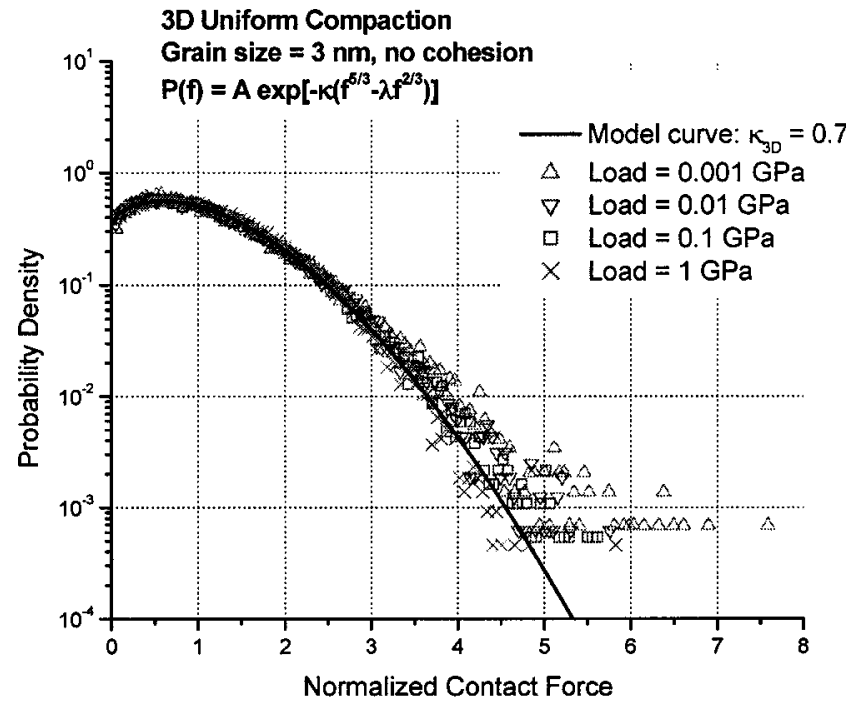

(a)

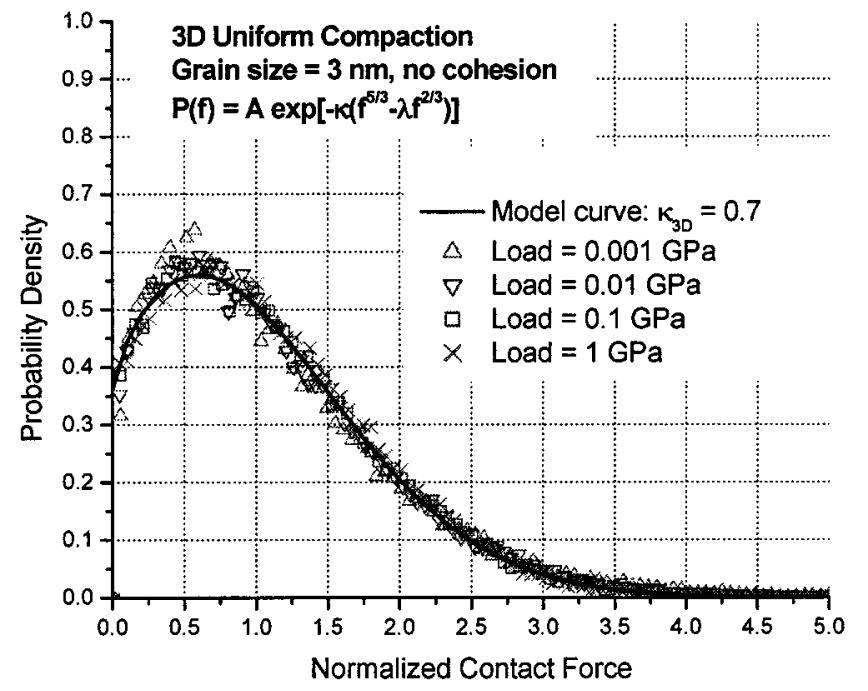

(b)

FIG. 3. Computer-simulated force distributions in 3D amorphous packings (same data from Fig. 1). (a) Logarithmic scale, (b) linear scale for the probability axis. The normalized force in the abscissa is $\langle f\rangle=f / \hat{f}$.

ancy at large forces under weak pressures is due to an intrinsic deficiency in using the Shannon entropy as suggested by Metzger or just a result of numerical errors in the simulation. The 3D calculations involved many more intergranular contacts than the $2 \mathrm{D}$ and therefore the numerical uncertainties are higher.

In the 2D Hertzian contact situation, as discussed above, the results due to the mean-strain constraint are the same as those from the mean-force constraint; i.e., Fig. 3 in Ref. [2] still stands. In Ref. [2], the smallest pressure used (0.0001 unit) corresponds to a mean force of $2.67 \times 10^{-4}$ unit, which leads to a mean strain of $0.0028 \%$. The deformation is already very small but the predicted Gaussian form still fits the simulated results very well (see Fig. 4). To see the effects of even smaller strains, two new calculations have been performed at 0.00001 and 0.000001 unit of applied pressure, 


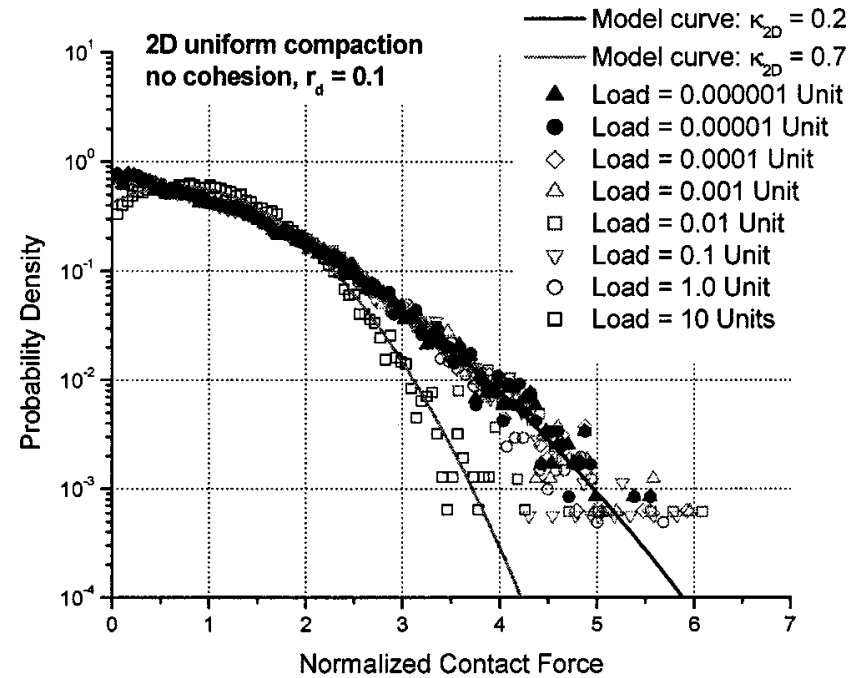

FIG. 4. Computer-simulated force distributions in 2D amorphous packings (original Fig. 3 of Ref. [2] with new results for 0.00001 and 0.000001 unit of load added).

corresponding to mean strains of $0.00028 \%$ and $0.000028 \%$, respectively. The results are shown in Fig. 4 and they are found to lie on top of the 0.0001 unit curve; i.e., they can still be fitted very accurately by a Gaussian curve. Hence, there is no indication that the $2 \mathrm{D}$ force distribution at vanishing pressures deviates from the Gaussian form predicted by Eq. (8) of Ref. [2].

\section{STATIC DETERMINACY VS INDETERMINACY}

Metzger argues that the force distributions resulting from different force laws must approach a universal form in the limit of infinite grain rigidity. In the special case of the Hookean force law, this conclusion leads to a dilemma situation which is explained here. A packing in which the contacts obey a Hookean force law is in fact a linear elastic structure. According to the principle of superposition in linear elasticity, the internal forces in such a structure must be proportional to the applied loads at the boundaries. Hence if we let the applied load (hydrostatic pressure in this case) approach zero, corresponding to letting the granular rigidity approach infinity, the forces cannot exhibit any odd behavior other than proportionality with the applied load. Thus, as long as the structure remains the same-and it will be so if the load range concerned is small- the normalized force distribution $P(\langle f\rangle)$ cannot change as the applied pressure tends to zero. Therefore, if one accepts the force distribution to be Gaussian at some small but finite strain, such as those shown in Fig. 4, the same $P(\langle f\rangle)$ will be maintained if the strain tends to zero from this strain. In the 2D simulations in Ref. [2] and the new results in Fig. 4 here, the structure of the packing is constant as long as the applied load is smaller than $\sim 0.1$ unit (see also Fig. 4 of Ref. [2]). Thus, the $P(\langle f\rangle)$ at vanishing pressures must be the same as the $P(\langle f\rangle)$ at, for example, 0.1 unit, 0.01 unit, 0.001 unit, and so on. The new results in Fig. 4 here confirm this. However, Metzger correctly pointed out that at infinite grain rigidity, the problem is statically determinate and so no force law is required to solve the problem (or the problem does not involve strain), while my argument here is for a statically indeterminate problem which requires knowledge of the interaction force law for a solution. It is unlikely that the Gaussian distribution valid for finite strains in the Hookean force-law situation is in fact the "universal" distribution Metzger proposed for statically determinate packings irrespective of force law. Now it may be possible that the zero-load limit of the "indeterminate" formulation does not match the solution of the "determinate" formulation of the same packing configuration. If this is the case, this will be an interesting dilemma for some future work to resolve. However, in actual experiments strains rather than forces are measured, and also in all the simulated results in the literature, force laws were prescribed. Thus when these literature results are discussed, one is referring to the "indeterminate" context.

\section{UNIFORMITY OF PHASE SPACE}

Metzger's main point in his Comment is that he challenges the use of the uniform-phase-space assumption for the granular packing situation. He illustrates the unfavorable biasing of weak forces by a special geometry in which two forces are aligned. In my opinion, to analytically prove the nonuniformity of the phase space of force is as difficult as to prove the uniformity of it. Any thermodynamic model can only treat equilibrium in a global scale but not equilibrium in a local scale, which must also be satisfied. On the other hand, to prove or disprove the uniformity of phase space, a full solution to the local equilibrium problem is needed. However, an analytical solution to the local problem is intractable without making assumptions but these themselves are difficult to justify. For example, the approximate $q$ models assume that the structure is regular but force transmission is random, and these two are self-contradictory. In Metzger's model shown in Fig. 1 of his Comment, forces $f_{1}$ and $f_{3}$ are assumed to be aligned, and in a random packing it is impossible that each grain has two aligning forces. Therefore, like the prediction of the $q$ model, Metzger's Eqs. (8) and (9) in his Comment can at best be regarded as approximate force distributions because they are based on unrealistic features of the random packing. They therefore should not be treated as serious evidence against the Gaussian distribution or in support of the exponential tail.

Whether the phase space of force in a stressed packing is uniform or not therefore remains as an open question. Certainly, the quality of fit of the predicted distributions to the simulated ones as evident in Figs. 3 and 4 indicates that the phase space of force is at least approximately uniform if not fully uniform.

To conclude, the observed discrepancy between theory and simulations in my original work can be removed by choosing a more realistic constraint for the free energy minimization. In the situation of Hookean contacts at least, there is no evidence to indicate that the force distribution would deviate from the Gaussian form at vanishing strains. Metzger's argument about the noninvolvement of the force law in the statically determinate situation points to an inter- 
esting dilemma against the superposition principle in linear elasticity, unless the Gaussian form turns out to be the universal distribution in statically determinate packings. Finally, Metzger's comment that the entropy approach does not represent local equilibrium is valid, but a convincing localequilibrium model free of unrealistic constraints is yet to be proposed.

\section{ACKNOWLEDGMENTS}

This work is supported by a research grant from the University Research Committee of the University of Hong Kong (Project No. 10204222.16180.14500.323.01). I am also grateful to P.T. Metzger for bringing up some interesting points for discussion.
[1] P. T. Metzger, previous paper, Phys. Rev. E 69, 053301 (2004).
[2] A. H. W. Ngan, Phys. Rev. E 68, 011301 (2003). 